Отсюда последовательно находим:

$$
\left.\begin{array}{l}
y_{1}=\frac{b_{1}}{t_{11}} \\
y_{i}=\frac{b_{i}-\sum_{k=1}^{i-1} t_{k i} y_{k}}{t_{i i}} \quad(i>1)
\end{array}\right\}
$$

и

$$
\left.\begin{array}{rl}
x_{n} & =\frac{y_{n}}{t_{n n}} \\
x_{i}= & y_{i}-\sum_{k=i+1}^{n} t_{i k} x_{k} \\
t_{i i}
\end{array}\right\}
$$

Изложенный способ решения линейной системы носит название метода квадратных корней.

$$
* * *
$$

1. Бахвалов Н.С., Жидков Н.П., Кобельков Г.М. Москва, Наука, Численные методы, Главная редакция физ. мат. литературы, 2008.

2. Демидович Б.П., Марон И.А. Основы вычислительной математики. Учебное пособие, Санкт Петербург - Москва - Краснодар, 2009.

3. ДемидовичБ.П Марон И.А, Шувалова Э.З. Численные методы анализа, Гос изд-во физ-мат. Лит., г. Москва 2007.

4. Курош А.Г., Курс высшей алгебры: Учебник. 16-изд., Изд. «Лань»; М.: Изд. «Физматкнига», 2010. $-432 \mathrm{c}$.

5. Токочаков В.И. Практическое пособие по теме "Решение систем алгебраических и дифференциальных уравнений в среде MathCadWindows" для студентов всех специальностей дневного и заочного отделений. - Гомель: ГГТУ, 2011.

\title{
Макарова М.В.
}

Построение гарантирующих управлений, как способ обеспечения надежности объектов при моделировании

Санкт-Петербургский государственный университет аэрокосмического приборостроения

doi: 10.18411/lj-06-2020-54

(Россия, Санкт-Петербург)

idsp: ljournal-06-2020-54

\section{Аннотация}

Исследуются объекты, математическими моделями которых являются линейные дифференциальные уравнения с постоянными коэффициентами. На эти объекты действуют возмущающие силы, которые имеют случайный характер. Известны их корреляционные функции и спектральные плотности мощности. Даётся алгоритм построения гарантирующего управления, которое обеспечивает надёжность рассматриваемых объектов. 
Ключевые слова: математическая модель, линейные дифференциальные уравнения, возмущающая сила, случайная функция, корреляционная функция, спектральная плотность мощности, линейный регулятор, устойчивость движения, оптимальное управление.

\section{Abstract}

Are investigated the objects, mathematical models of which are linear differential equations with the constant coefficients. On these objects the perturbing forces, which have random nature, act. Their correlation functions and spectral power densities are known. Is given the algorithm of the construction of guaranteeing control, which ensures the reliability of the objects in question.

Keywords: mathematical model, linear differential equations, perturbing force, random function, correlation function, spectral power density, proportional regulator, stability of motion, optimal control.

Настоящая статья посвящена исследованию объектов, математическими моделями которых служат линейные дифференциальные уравнения с постоянными коэффициентами. На исследуемые объекты действуют возмущающие силы случайного характера, относительно которых нам известны их корреляционные функции и спектральные плотности мощности. Для уменьшения влияния возмущающих сил используются линейные регуляторы с обратной связью.

Математические модели подобных объектов имеют вид:

$$
A(D) x=B(D) u+\varphi(t)
$$

где $x$ - управляемая величина, $u$ - управляющее воздействие, $A(D)$ и $B(D)$ - полиномы от оператора дифференцирования $D=\frac{d}{d t}, \varphi(t)$ - случайная функция времени, относительно которой известна её корреляционная функция $K_{\varphi}(\tau)$, называемая ещё автокорреляционной функцией:

$$
K_{\varphi}(\tau)=\lim _{T \rightarrow \infty} \frac{1}{T} \int_{0}^{T} \varphi(t) \varphi(t+\tau) d t .
$$

Спектральной плотностью мощности (далее по тексту - спектр) называется следующее косинус-преобразование Фурье корреляционной функции:

$$
S_{\varphi}(\omega)=\frac{2}{\pi} \int_{0}^{\infty} K_{\varphi}(\tau) \cos \omega \tau d \tau
$$

Значение спектра заключается в том, что он позволяет легко вычислить как средний квадрат самой функции $\varphi(t)$ :

$$
<\varphi^{2}>=\lim _{T \rightarrow \infty} \frac{1}{T} \int_{0}^{T} \varphi^{2}(t) d t=\int_{0}^{\infty} S_{\varphi}(\omega) d \omega,
$$

так и, самое главное, средний квадрат решения линейного дифференциального уравнения с постоянными коэффициентами, в правой части которого присутствует случайная функция $\varphi(t)$. Так, если

To

$$
\begin{gathered}
A(D) x=B(D) \varphi(t), \\
<x^{2}>=\int_{0}^{\infty} \frac{|B(j \omega)|^{2}}{|A(j \omega)|^{2}} S_{\varphi}(\omega) d \omega .
\end{gathered}
$$

Вопросы использования спектров для реализации наилучшего (оптимального) управления при не полностью известных и случайных возмущающих силах более подробно освещены в монографии [1].

Пример: движение корабля по курсу описывается уравнением

$$
A(D) x=u+\varphi(t)
$$


где $u$ - это управляющее воздействие корабельного руля. Тогда, например, для танкеров серии «Казбек» имеем

$$
A(D)=T_{1}^{2} D^{2}+T_{2} D
$$

причём, $\mathrm{T}_{1}=690 \mathrm{ce \kappa}^{2}, \mathrm{~T}_{2}=17,2$ cек, а возмущающее воздействие от морского волнения $\varphi(t)$ имеет корреляционную функцию и спектр хорошо аппроксимируемые известными формулами Рахманини-Фирсова:

$$
\left.\begin{array}{c}
K_{\varphi}(\tau)=<\varphi^{2}>\cdot e^{-\alpha \tau}\left(\cos \beta \tau+\frac{\alpha}{\beta} \sin \beta \tau\right) \\
S_{\varphi}(\omega)=<\varphi^{2}>\cdot \frac{4 \alpha}{\pi} \cdot \frac{\alpha^{2}+\beta^{2}}{\left(\alpha^{2}+\beta^{2}+\omega^{2}\right)^{2}-4 \beta^{2} \omega^{2}}
\end{array}\right\}
$$

где $\alpha$ и $\beta$ имеют размерность $1 /$ сек и для развитого морского волнения отношение $\alpha / \beta$ колеблется обычно в пределах $0,15 \leq \alpha / \beta \leq 0,25$. При таких соотношениях спектр имеет острый максимум вблизи частоты $\omega=\sqrt{\beta^{2}-\alpha^{2}}$ и основная часть энергии возмущающего воздействия сосредоточена вблизи частот $\omega \approx \beta$. Величина $\beta$ зависит от района плавания и интенсивности волнения и может изменяться в широких пределах от 0,5 до 1,7 сек $^{-1}$.

Вычисляя $\left\langle x^{2}\right\rangle$ для танкеров серии «Казбек» с использованием формул (8) и (9), замечаем, что при $u=0$ интеграл (6) в данном случае расходится. Это подтверждает еще раз, что движение корабля на волнении без руля, без управляющего воздействия не устойчиво.

Для обеспечения устойчивости движения и малых отклонений корабля от заданного курса применяют (используя руль) управление, формируемое чаще всего линейным регулятором вида

$$
u=-\frac{W_{1}(D)}{W_{2}(D)} x
$$

где $W_{1}(D)$ и $W_{2}(D)$ - полиномы от оператора дифференцирования $D=\frac{d}{d t}$. Для упрощения дальнейших формул будем записывать линейный регулятор (10) в виде $u=W(D)$, имея в виду, что $W(D)$ может быть и рациональной дробью. Уравнение движения корабля по курсу с учётом как возмущающих сил, так и корабельного руля, будет иметь вид:

$$
A(D) x+W(D) x=\varphi(t)
$$

и средний квадрат $<x^{2}>$ отклонений корабля от курса будет равен

$$
<x^{2}>=\int_{0}^{\infty}|A(j \omega)+W(j \omega)|^{2} \cdot S_{\varphi}(\omega) d \omega .
$$

За счёт правильного выбора функции $W(D)$ в математической модели движения корабля и последующей реализации этой функции в работающем рулевом устройстве можно обеспечить устойчивое движение по курсу с малыми отклонениями от заданного курса и наименьшей потерей скорости при рыскании корабля на курсе. Реализующий наилучшим образом все эти требования регулятор называют оптимальным регулятором и он зависит, разумеется, от спектра возмущающих сил. Для спектров вида (9) он зависит от коэффициентов $\alpha$ и $\beta$. Примеры этих зависимостей и методы построения и реализации оптимальных регуляторов приведены в публикации [2].

Пояснив на простом примере управления по курсу особенности управления при случайных воздействиях, перейдём к общей проблеме управления для объектов, математическими моделями которых являются уравнения (1) при квадратичных критериях качества.

Если объект управления (1) замкнут регулятором с обратной связью $u=$ $-W(D) x$, то средний квадрат регулируемой величины $x$ равен интегралу 


$$
<x^{2}>=\int_{0}^{\infty}|A(j \omega)+B(j \omega) W(j \omega)|^{2} \cdot S_{\varphi}(\omega) d \omega,
$$

т.е. равен интегралу от произведения двух функций от переменной $\omega$ (частоты). Первая функция - это квадрат модуля передаточной функции объекта управления с учётом управления $u=-W(D) x$, а вторая - это спектр $S_{\varphi}(\omega)$ возмущающих сил. Если этот спектр известен, то хорошо известны и описанные в многочисленных руководствах методы, позволяющие вычислить и реализовать управление, наилучшее (оптимальное) для данного спектра. История вопроса и различные подходы к оптимизации управления изложены в очерке [3].

Оптимальное управление оптимально для конкретного спектра возмущающих воздействий, но возможны такие условия работы управляемого объекта, когда ему приходится сталкиваться с различными возмущающими воздействиями и нужно, чтобы единый регулятор $u=-W(D)$ обеспечил для него хорошее качество работы для любого из возможных спектров. Характерным примером является управление кораблём по курсу в различных погодных условиях, а, значит, и при различных спектрах с которыми придётся встретиться в ходе плавания.

Дойл, Френсис и ряд других авторов сначала в серии журнальных статей, затем, в монографиях [4] и [5] предложили следующий подход к решению данной задачи. Регулятор $u=-W(D) x$ следует подбирать так, чтобы частотная характеристика замкнутой системы (объект управления замкнутый регулятором) имела бы возможно более равномерную частотную характеристику в интересующем нас интервале частот. Если эта характеристика идеально равномерна, то при любом спектре $S_{\varphi}(\omega)$ (с нормирующим условием $\left.\int_{0}^{\infty} S_{\varphi}(\omega) d \omega=1\right)$ возмущающего воздействия средний квадрат регулируемой величины будет постоянной величиной, не зависящей от спектра. Если частотная характеристика примерно равномерна, то отклонения $<x^{2}>$ от постоянной величины для всех $S_{\varphi}(\omega)$ будут не велики.

Для обеспечения постоянства частотной характеристики замкнутой системы и соответствующего выбора регулятора $u=-W(D) x$ была предложена довольно сложная методика, основанная на известной теореме Пика-Неванлинна. Эта методика была подхвачена российскими учёными (см. обзорные статьи [6] и [7] и монографию [8]). Заметим, что символы $\mathrm{H}^{\infty}$ или $\mathrm{H}_{\infty}$ в заглавиях и в тексте этих статей и книги связаны с фамилией математика Харди (Hardy) с именем которого связаны понятия «пространство Харди» с соответствующей метрикой.

Однако более подробный анализ показывает, что обеспечение возможно более равномерной частотной характеристики не является наилучшим решением.

Рассмотрим объект управления

$$
(D+1) x=u+\varphi(t)
$$

с критерием качества $J=<x^{2}>+<u^{2}>$, учитывающим расходы на управление. Для объекта управления

$$
<\varphi^{2}>=\int_{0}^{\infty} S_{\varphi}(\omega) d \omega=1
$$

выбранный критерий качества зависит линейно от среднего квадрата возмущающего воздействия и более сложно зависит от его спектра. В дальнейшем возмущающее воздействие будем нормировать, т.е. считать, что $\left\langle\varphi^{2}\right\rangle=1$. Пересчёт на другие значения $<\varphi^{2}>$ затруднений не представляет.

Для такого простого объекта как (13) сразу видно, что наиболее опасным возмущающим воздействием является постоянная сила $\varphi(t)=1$, для которой к минимуму критерия качества приводит управление 


$$
u=-k x \text {, }
$$

при котором уравнение движения объекта приобретает вид

$$
(D+1+k) x=\varphi(t),
$$

и это позволяет легко вычислить, что

$$
\begin{gathered}
<x^{2}>=\int_{0}^{\infty} S_{\varphi} \cdot \frac{d \omega}{(1+k)^{2}+\omega^{2}} \leq \frac{1}{(1+k)^{2}} \\
<u^{2}>=k^{2} \cdot<x^{2}>\leq \frac{k^{2}}{(1+k)^{2}}
\end{gathered}
$$

Получаем оценку критерия качества

$$
J=\left\langle x^{2}>+<u^{2}>=\int_{0}^{\infty} S^{\prime}{ }_{\varphi}(\omega) \cdot \frac{\left(1+k^{2}\right) d \omega}{(1+k)^{2}+\omega^{2}} \leq \frac{1+k^{2}}{(1+k)^{2}}\right.
$$

При наиболее опасном возмущающем воздействии $\varphi=1$ (спектром которого является известная $\delta$-функция Дирака $\delta(0)$ в начале координат) значение критерия качества наибольшее и равно $\frac{1+k^{2}}{(1+k)^{2}}$, а для всех других спектров критерий качества меньше своего максимального значения. Это означает, что управление (15) является гарантирующим - оно гарантирует, что для любых спектров критерий качества будет не больше, а чаще всего меньше, легко вычисляемого гарантированного значения.

Гарантирующее управление, которое для спектров обеспечивает критерию качества значительно меньшую величину, чем для спектра наихудшего, обеспечивает для управляемого объекта лучший результат его работы, чем оптимизация по равномерно-частотным показателям, когда критерий качества для всех возможных спектров остаётся примерно постоянной величиной.

Первые примеры гарантирующих управлений и сам термин «гарантирующее управление» были приведены в монографии [2, с.115-120]. Многочисленные примеры и методы синтеза гарантирующих управлений приведены в публикации [9]. Однако, после появления в 1981 году первой статьи Зеймса по «равномерно-частотной оптимизации» (содержание статьи приведено в обзоре [6]) хлынул целый вал статей и, затем, монографий по этой тематике, часто также называемой «методикой $\mathrm{H}^{\infty}$ управления» или « $\mathrm{H}^{\infty}$ теорией оптимизации». На фоне этого бурного потока работ по « $\mathrm{H}^{\infty}$ оптимизации» почти совершенно не замеченными остались публикации по гарантирующему управлению, несмотря на все их преимущества перед « $\mathrm{H}^{\infty}$ управлением». История вопроса изложена в монографии [3, с.166-182].

Методика синтеза гарантирующего регулятора не является сложной. Для объектов управления, математическими моделями которых служат уравнения (1), достаточно выполнить следующие операции:

1. Сначала вычислим значение $\omega=\beta$, при котором достигает наименьшего значения функция

$$
M=|A(j \omega)|^{2}+m^{2}|B(j \omega)|^{2} .
$$

Наиболее неблагоприятным спектром для объекта (1) в этом случае является спектр в виде $\delta$-функции Дирака $\delta(\beta)$ с аргументом $\beta$. Если $\beta=0$, то в этом случае спектр $S_{\varphi}(\omega)=\delta(0)$ соответствует возмущающему воздействию в виде постоянной силы $\varphi(t)=1$. Если $\beta \neq 0$, то спектру $S_{\varphi}(\omega)=\delta(\beta)$ соответствует гармоническое колебание с частотой $\beta$, т.е. в этом случае $\varphi(t)=\sin \beta t$.

2. На втором этапе синтезируем регулятор, оптимальный для возмущающего воздействия $\varphi(t)=1$, (если минимум функции (19) достигается при $\omega=0)$, либо оптимальный для $\varphi(t)=\sin \beta t$, (если этот минимум достигается при $\omega=\beta)$. 
3. $\quad$ Далее, для найденного оптимального регулятора $u=-W(D) x$ проверяем поведение функции

$$
|A(j \omega)+B(j \omega) W(j \omega)|^{2}
$$

Если для любого $\omega$ эта функция не больше (а еще лучше, если меньше), чем при $\omega=\beta$, то синтезированный регулятор является гарантирующим.

Поясним второй этап. Регулятор, оптимальный для возмущающих воздействий $\varphi(t)=1$ или $\varphi(t)=\sin \beta t$ со спектрами $\delta(0)$ и $\delta(\beta)$ не может быть синтезирован по общим методикам синтеза оптимальных регуляторов, изложенным, например, в издании [1], поскольку все эти методики предполагают запись спектра в виде рациональной дроби. Однако, $\delta$-функции Дирака $\delta(\omega=0)$ и $\delta(\omega=\beta)$ можно рассматривать как пределы многих рациональных дробей.

Пример. Спектры в виде рациональных дробей

$$
\begin{aligned}
& S_{1 \varphi}(\omega)=\frac{2}{\pi} \cdot \frac{\alpha}{\alpha^{2}+\omega^{2}} \\
& S_{2 \varphi}(\omega)=\frac{4}{\pi} \cdot \frac{\alpha^{3}}{\left(\alpha^{2}+\omega^{2}\right)^{2}}
\end{aligned}
$$

при $\alpha \rightarrow 0$ переходят в одну и ту же $\delta$-функцию Дирака $\delta(0)$. Если необходимо для объекта управления (13) с критерием качества $\left.J=3<x^{2}\right\rangle+\left\langle u^{2}\right\rangle$ построить регулятор, оптимальный для $\delta$-функции Дирака $\delta(0)$, то можно построить регулятор, оптимальный для спектра (21) и он будет иметь вид

$$
u=-\left(\frac{1}{1+\alpha} D+\frac{3+\alpha}{1+\alpha}\right) x
$$

В пределе при $\alpha \rightarrow 0$ он перейдёт в регулятор

оптимальный для $\delta$-функции Дирака $\delta(0)$.

$$
u=-(D+3) x
$$

Если взять за основу при проектировании регулятора вместо спектра (21) спектр (22), то получим регулятор

$$
u=-\left[\frac{6+6 \alpha+\alpha^{2}+(3+2 \alpha) D+D^{2}}{2+2 \alpha+\alpha^{2}-D}\right] x,
$$

который в пределе, при $\alpha \rightarrow 0$, перейдёт в регулятор

$$
u=-\left[\frac{6+3 D+D^{2}}{2-D}\right] x .
$$

И регулятор (24) и регулятор (26) в равной мере являются гарантирующими, но регулятор (24) проще и, поэтому, более удобен.

Любопытно, что существует ещё более простой регулятор $u=-3 x$, тоже являющийся гарантирующим. Вообще, для объектов управления, у которых функция (19) достигает наименьшего значения при $\omega=0$ (а таких объектов много), часто имеют простые гарантирующие регуляторы вида $u=-k x$. Возможно, что именно этим объясняется широкое распространение простых пропорциональных регуляторов $u=-$ $k x$ до самого последнего времени.

Если наименьшее значение функции (19) достигается при $\omega=\beta \neq 0$, то в этом случае надо искать регулятор, оптимальный для дельты-функции Дирака $\delta(\omega=\beta)$. Для того, чтобы и в этом случае использовать стандартную методику, можно искать регулятор оптимальный, например, для спектра (9), а, затем, перейти к пределу при $\alpha \rightarrow 0$. Если же вместо спектра (9) использовать похожий спектр

$$
S_{\varphi}=\frac{4 \alpha}{\pi} \cdot \frac{\left(\alpha^{2}+\beta^{2}\right)\left(1+k^{2} \omega^{2}\right)}{\left(\alpha^{2}+\beta^{2}+\omega^{2}\right)^{2}-4 \beta^{2} \omega^{2}}
$$

то управляемый объект (как показано в монографии [1]), получит дополнительное свойство сохранения устойчивости при малых вариациях параметров. 
Вообще, наличие не одного, а многих гарантирующих регуляторов позволяет выбрать из них наиболее простой и удобный, а также удовлетворяющий дополнительным требованиям к системе управления. Поэтому гарантирующие регуляторы могут иметь весьма широкую область применения.

$$
* * *
$$

4. Петров Ю.П. Синтез оптимальных систем управления при неполностью известных возмущающих силах. - Л.: Изд-во ЛГУ, 1987. - 289с.

5. Петров Ю.П. Оптимизация управляемых систем, испытывающих воздействие ветра и морского волнения. - Л.: Судостроение, 1973. - 216с.

6. Петров Ю.П. Очерки истории теории управления. - СПб.: БХВ-Петербург, 2007. - 266с.

7. J.Doyle, B.Fransis, A.Tannenbaum. Control Theory. New York: Macmillan Publ.Co., 1992. - 227p.

8. Fransis B.A. A Course in H_oControl Theory. Berlin: Springer Verlag, 1987. - 156p.

9. Барабанов А.Е., Первозванский А.А. Оптимизация по равномерно-частотным показателям $\left(\mathrm{H}^{\wedge} \infty\right.$ теория). //Автоматика и телемеханика, 1992. №9. - С.3-32.

10. Себряков Г.Г., Семенов А.В. Методы $\mathrm{H}^{\wedge} \infty$ теории управления. //Техническая кибернетика, 1989. №2. - С.3-16.

11. Веремей Е.И. Линейные системы с обратной связью. СПб.: Лань, 2013. - 442c.

12. Петров Ю.П. Новые главы теории управления и компьютерных вычислений. - СПб.: БХВПетербург, 2004. - 191c. 